\title{
OCORRÊNCIA CLÍNICA DE INTERAÇÕES MEDICAMENTOSAS EM PRESCRIÇÕES DE PACIENTES COM SUSPEITA DE REAÇÃO ADVERSA INTERNADOS EM UM HOSPITAL NO INTERIOR DA BAHIA
}

\author{
OCCURRENCE OF CLINICAL DRUG INTERACTIONS IN PRESCRIPTIONS OF PATIENTS \\ SUSPECT OF ADVERSE REACTION HOSPITALIZED IN THE COUNTRYSIDE \\ OF THE STATE OF BAHIA, BRAZIL
}

\section{Suéllyn dos Santos Gonçalves ${ }^{a}$, Hosana Maria Santos Rodrigues ${ }^{b}$, Inocêncio Silva de Jesus ${ }^{c}$, Jose Ailton Oliveira Carneiro ${ }^{\mathrm{d}}$, Gisele da Silveira Lemos ${ }^{\mathrm{e}}$ \\ asuellyn.jac@hotmail.com, bnana_rodrigues_4@hotmail.com, cinocencio_silva@hotmail.com, dhitoef@yahoo.com.br, egisalemos@ig.com.br Universidade Estadual do Sudoeste da Bahia - Jequié (BA), Brasil}

Data de recebimento do artigo: 01/12/2014 Data de aceite do artigo: 14/01/2016

\section{RESUMO}

As interaçóes medicamentosas constituem um dos graves problemas de saúde pública e estão relacionadas a internaçôes hospitalares e aumento de gastos com médicos. O objetivo deste trabalho foi avaliar a ocorrência clínica de interaçóes medicamentosas em pacientes com suspeita de reaçôes adversas internados em um hospital. Realizou-se um estudo de segmento com acompanhamento prospectivo náo concorrente de 31 prontuários. Para análise, foram utilizados o banco de dados Micromedex e as classificaçóes ATC e CID10. Observou-se que $61,3 \%$ dos pacientes eram do sexo masculino com média de idade de 56 $\pm 23,8$ anos. Foram analisadas 858 prescriçóes, sendo encontradas 1507 interaçóes medicamentosas, das quais 35\% de gravidade maior e 33\% de gravidade moderada. Do total de interaçóes medicamentosas, 53\% puderam ser observadas clinicamente - dessas, 11,08\% causaram reaçôes adversas. Para verificar a associação entre ocorrência de interaçóes medicamentosas e os fatores sexo, idade e número de medicamentos prescritos, foi utilizada técnica de regressáo logística binária e calculado odds ratio, com intervalos de confiança de 95\% (IC 95\%). Pela análise dos resultados, pôde-se concluir que o número de medicamentos, a idade e o sexo estão diretamente associados à ocorrência de interaçóes medicamentosas; conhecendo essas variáveis, podem-se minimizar os danos e riscos à saúde.

Palavras-chave: Prescriçóes; interaçóes de medicamentos; pacientes internados.

\section{ABSTRACT}

Drug interactions (DI) are a significant public health problem, and are associated with increased health care costs. The objective of this study was to evaluate the clinical occurrence of DI in patients with suspected adverse reactions in a hospital. We conducted a segment study with non-concurrent prospective follow-up of 31 medical records. In the analysis of the data was used the Micromedex ${ }^{\oplus}$ database and ICD10 and ATC classifications. It was observed that $61.3 \%$ of patients were male with a mean age of $5623.8 \pm$ years. We analyzed 858 prescriptions andfound 1507 drug interactions. Of these, 35\% were highly severe and $33 \%$ were moderately severe. From the overall DI, $53.0 \%$ were clinically observed - and $11.08 \%$ caused adverse reactions. To verify the association between the occurrence of DI and sex, age and number of drugs prescribed, it was used technical binary logistic regression and the odds ratio (OR) were calculated with confidence intervals of $95 \%$ (95\% CI). By the analysis of the results, it was concluded that the amount of drugs, age and sex are directly associated with the occurrence of DI and knowing these variables can minimize damage and health risks.

Keywords: Prescriptions; drug interactions; inpatients. 


\section{Introdução}

O uso concomitante de vários medicamentos é comum na prática clínica e está inerentemente relacionado ao risco de interaçóes medicamentosas (IM). A politerapia é justificável quando permite obter efeito terapêutico sinérgico, aumentando a eficácia do tratamento, ou para a terapia de múltiplas doenças coexistentes ${ }^{1}-$ porém, essa prática deve ser realizada com cuidado, visto que a utilização de vários medicamentos pode acarretar efeitos indesejáveis. As IM são um evento clínico em que os efeitos de um fármaco são alterados pela presença de outro fármaco, alimento, bebida, ou de algum agente químico ou ambiental, constituindo causa comum de eventos adversos ${ }^{2}$. As IM podem ser um acontecimento perigoso, visto que a toxicidade de um medicamento pode ser alterada na presença de outros, trazendo danos à saúde do paciente 3 .

As IM são um dos problemas graves de saúde pública e estáo relacionadas a internaçóes hospitalares e aumento de gastos em saúde ${ }^{4}$. Estima-se que 3,8\% das internaçóes por eventos adversos a medicamentos são decorrentes de $\mathrm{IM}^{5}$, sendo que 68-70\% das IM demandam cuidados médicos e 1-2\% colocam em risco a vida do paciente ${ }^{6}$. Avalia-se que há $30 \%$ de chance de ocorrência de IM em pacientes que utilizam dois ou mais medicamentos, e esse risco aumenta em $100 \%$ quando utilizam oito ou mais, o que certifica que a taxa de ocorrência de IM sobe paralelamente à elevação do número de medicamentos ${ }^{7}$.

Apesar de esse ser, atualmente, um dos temas mais importantes da farmacologia clínica na prática dos profissionais da saúde, a frequência de IM clinicamente importantes é pouco descrita na literatura ${ }^{8}$; e, como a maioria dos pacientes hospitalizados utiliza muitos medicamentos, a dimensão do problema é nitidamente relevante ${ }^{9}$. É necessária a implementação de medidas que visem à redução da ocorrência de IM, tendo como base a avaliação das prescrições médicas e a realização de estudos de utilização de medicamentos, para embasar e contribuir no estabelecimento dessa estratégia de ação, assegurando alguma qualidade de vida ao paciente hospitalizado. Em decorrência da posição ocupada no sistema de utilização de medicamentos, o profissional farmacêutico representa um elo entre o processo de distribuição e o de utilização deles, além de ter um papel importante na identificação e correção de problemas possíveis e reais que envolvam medicamentos, proporcionando benefícios para o paciente ${ }^{10,11,12}$. Dessa forma, faz-se necessária a introdução de um método que vise à prescrição racional, sendo realizadas análises críticas de risco e benefício das politerapias propostas e de prescriçôes de medicamentos antes da administração ao paciente $^{12}$.
Diante disso, o objetivo deste trabalho foi avaliar a ocorrência clínica de IM em prescriçóes de pacientes com suspeita de reaçóes adversas internados em um hospital do interior da Bahia.

\section{Metodologia}

Realizou-se um estudo de segmento com acompanhamento prospectivo não concorrente, de âmbito regional, em 31 pacientes com suspeita de reação adversa internados nas diversas especialidades - clínica médica, clínica cirúrgica, neurologia, pediatria e terapia intensiva - de um hospital regional de ensino. O hospital, que é vinculado à Secretaria Estadual de Saúde da Bahia e se localiza no municípi o de Jequié, presta atendimento a 180 leitos e atende uma população de cerca de 500 mil habitantes de 25 municípios pertencentes ao Núcleo Regional de Saúde Sul.

A coleta de dados foi realizada no período de setembro de 2013 a abril de 2014; analisaram-se 31 prontuários de pacientes que apresentaram reação adversa a medicamento durante o período de internação e que faziam uso de mais de dois medicamentos. Esses pacientes foram monitorados diariamente por meio de análise documental e investigação dos possíveis efeitos clínicos das IM com o auxílio do programa Micromedex ${ }^{\odot}$ Healthcare Series $2.0^{13}$ - ferramenta considerada padrão de excelência em informação clínica, trazendo informações sustentadas em revisões sistemáticas acerca de medicamentos -, classificando as IM quanto à gravidade (leve, moderada, grave e contraindicada), ao tempo de início (tardio ou rápido), ao mecanismo de ação e ao nível de evidência científica (boa, excelente, pobre, improvável ou desconhecida).

Aplicaram-se instrumentos de coleta para obter informaçôes relacionadas às características biodemográficas e sociais, sendo eles: ficha de coleta de medicamentos (dose, via de administração, duração do tratamento); desfecho do internamento e classificaçóes CID $10^{14}$ (Classificação Internacional de Doenças e Problemas Relacionados à Saúde) descritas com um código alfanumérico e publicadas pela Organização Mundial de Saúde (OMS) para padronizar e codificar as doenças; e ATC (Anatomical Therapeutic Chemical Classification Index), desenvolvido pelo Centro Colaborador de Metodologias Estatísticas de Medicamentos da OMS ${ }^{15}$, em que os fármacos são classificados em grupos e subgrupos e obedecem a cinco níveis, de acordo com os órgãos e sistemas de atuação, bem como suas propriedades químicas, terapêuticas e farmacológicas.

Foram considerados como critérios de inclusão que todos os pacientes apresentassem suspeita de reaçóes adversas a medicamentos (RAM), tivessem na prescrição 
mais de dois fármacos e concordassem em participar da pesquisa, preenchendo o TCLE. Os que não apresentavam suspeita de RAM, que não tinham mais de dois medicamentos na prescrição ou não concordaram em preencher o TCLE não foram incluídos na pesquisa. A identificaçáo das RAMs aconteceu simultaneamente em um estudo em paralelo, com o auxílio de rastreadores; realizou-se a análise de causalidade para suspeitas de RAM de acordo com o Algoritmo de Naranjo e da OMS.

Utilizou-se o programa Microsoft Excel $^{\circledR} 2010$ como banco de dados para transcrever as informaçóes extraídas dos prontuários e das prescrições médicas durante o período de internação. O processamento e a análise dos dados foram realizados utilizando o programa SPSS versão 21.0. Para análise dos dados, procedeu-se a distribuição de frequências das variáveis estudadas, organizadas em tabelas e gráficos. Para a análise descritiva das características dos dados, calcularam-se as frequências absolutas e relativas. A associação entre faixa etária, sexo e número de medicamentos por prescrição com ocorrência de IM foi feita por meio da técnica de regressão logística binária. Foram calculados odds ratio (OR) com os seus respectivos intervalos de confiança de $95 \%$ (IC 95\%). Em todas as análises o nível de significância adotado foi de $5 \%(\mathrm{p}<0,05)$. Os dados foram analisados no IBM SPSS Statistics for Windows (IBM SPSS. 21.0, 2012, Armonk, NY: IBM Corp.).

Este estudo obedeceu à Resolução número 466 de 12 de dezembro de 2012 do Conselho Nacional de Saúde do Ministério da Saúde. A pesquisa foi aprovada pelo Comitê de Ética em Pesquisa da Universidade Estadual do Sudoeste da Bahia, CAAE n ${ }^{\circ}$ 19098713.8.0000.0055.

\section{Resultados e discussão}

No estudo, foram analisadas 858 prescriçóes de 31 pacientes, sendo que $61,3 \%$ eram do sexo masculino, com média de idade de $48,3 \pm 25,76$, e $38,7 \%$ do sexo feminino, com média de idade de $68,16 \pm 14,77$. Do total dos pacientes, 70,96\% eram pardos, $19,35 \%$ brancos e $9,67 \%$ negros, como pode ser visto na tabela 1 . A média geral de idade foi de $56 \pm 23,8$, com variação entre 11 e 87 anos, sendo que 56,06\% tinham mais de 60 anos. Segundo Malone et al. ${ }^{16}$, pessoas com mais de 60 anos são mais predispostas à ocorrência de IM, visto que esses indivíduos em sua maioria são portadores de doenças crônicas, o que acarreta necessidade de associaçáo de diversos medicamentos. $\mathrm{Na}$ amostra do estudo de Araujo et al. ${ }^{17}$ sobre readmissóes e óbitos pós-alta da unidade de terapia intensiva (UTI), 61,2\% eram do sexo masculino, e a média de idade foi de 53,69 $\pm 17,63$ anos. No estudo de Melo ${ }^{18} \mathrm{em}$ um hospital universitário da Universidade de São Paulo, a média de idade foi de $56,7 \pm 19,8$ anos, valores próximos aos encontrados neste estudo. A unidade de internamento mais prevalente foi a UTI, representando $70,8 \%$ das internaçôes, seguida de 12,8\% na Clínica Médica, 9,9\% na Clínica Cirúrgica, $3,22 \%$ na Pediatria e 3,22\% na Neurologia. Observouse que a média de internamento foi de $33,51 \pm 25,86$ dias, apresentando valores diferentes dos encontrados no estudo de Silva et al. ${ }^{19}(8,9 \pm 10,90)$ sobre fatores associados ao óbito e à readmissão em UTI. Essa diferença pode ter ocorrido pelo fato de alguns pacientes sofrerem transferência para outra unidade de internação, tendo sido contabilizado todo o período de internamento. O número de medicamentos variou de 2 a 23, com média de $8,66 \pm 4,37$ por prescrição, valor abaixo dos encontrados nos estudos de Rossignoli et al. $.^{20}-12,41 \pm 4,48$ na UTI de um Hospital Universitário em Marília - e de Hammes et al. ${ }^{21}$ em Joinville, que obteve a média de 13,10 $\pm 5,95$ de medicamentos por prescrição. Com relação ao cuidado, dos pacientes acompanhados $40 \%$ tiveram alta e os outros $60 \%$ foram a óbito.

nsiderando as informaçóes relacionadas aos diagnósticos dos pacientes com suspeita de reaçóes adversas segundo a CID10, foram prevalentes as CIDs primárias - lesão e outras consequências de causas externas (categoria $S$ ) -, seguidas de doenças do aparelho respiratório (categoria J) e circulatório (categoria I), representando $22,58 \%$ cada. A prevalência da CID primária, lesão e outras consequências de causas externas, pode ser justificada pelo fato de que o hospital situa-se às margens da BR 116, onde são frequentes os acidentes automobilísticos. No estudo de $\mathrm{Lima}^{22} \mathrm{em}$ um hospital no Ceará, $24 \%$ dos pacientes apresentaram doenças respiratórias e 24,9\% doenças circulatórias, resultados próximos aos encontrados neste estudo. Segundo Pincelli ${ }^{23}$, doenças respiratórias são causas frequentes de internamento em UTIs, destacando-se doença pulmonar obstrutiva crônica (DPOC) e pneumonia, que neste estudo representam $16,12 \%$. Das doenças circulatórias, a mais prevalente foi a hipertensão arterial sistêmica $(29,03 \%)$, valor próximo ao encontrado por Lima et al. ${ }^{22}$ (37\%) em seu estudo.

$\mathrm{Na}$ análise de associação medicamentosa com o auxílio do banco de dados Micromedex ${ }^{\ominus}$, foram encontradas 1.507 IM potenciais, das quais 72 tipos de IM diferentes, caracterizando média de 1,6 $6 \pm 1,62$ IM por prescrição. Rama et al. ${ }^{24}$, em seu estudo, encontraram média de 2,7 IM por prescrição, assim como $\mathrm{Melo}^{18} \mathrm{em}$ estudo de um hospital no sul da Índia, cuja média foi de 1,9 IM por prescrição. Na UTI, o paciente em estado crítico necessita de um atendimento complexo, sendo a utilizaçáo de vários medicamentos uma prática normal; portanto, a possibilidade da ocorrência de IM e do desenvolvimento de reaçóes adversas aumenta ${ }^{25}$. 
Tabela 1: Distribuição percentual de dados biodemográficos dos pacientes com suspeita de reações adversas internados no HGPV de setembro de 2013 a abril de 2014, Jequié (BA).

\begin{tabular}{lcc}
\multicolumn{1}{c}{ Características } & Fa $^{*}$ & Fr** $^{* *}$ \\
Sexo & & \\
\hline Feminino & 12 & $38,7 \%$ \\
\hline Masculino & 19 & $61,3 \%$ \\
\hline Cor & & \\
Pardo & 22 & $70,96 \%$ \\
\hline Branco & 6 & $19,35 \%$ \\
\hline Negro & 3 & $9,67 \%$ \\
Unidade de internamento & & \\
Unidade de terapia intensiva & 22 & $70,8 \%$ \\
\hline Clínica médica & 04 & $12,8 \%$ \\
\hline Clínica cirúrgica & 03 & $9,9 \%$ \\
\hline Neurologia & 01 & $3,22 \%$ \\
\hline Pediatria & 01 & $3,22 \%$ \\
Resultado do cuidado & & \\
\hline Óbito & 18 & $60 \%$ \\
\hline Alta hospitalar & 13 & $40 \%$ \\
\hline $\begin{array}{l}\text { Duraçáo do internamento } \pm \text { DP*** } \\
\text { Idade }\end{array}$ & & $33,51 \pm 25,86$ \\
\hline$<60$ anos & 13 & $41,93 \%$ \\
\hline 60 anos & 18 & $56,06 \%$ \\
\hline
\end{tabular}

Fonte: Pesquisa Direta.

*Fa: Frequência absoluta; **Fr: Frequência relativa.

***Média \pm desvio padrão.

Das IM identificadas, 35\% (532) foram de gravidade maior, 33\% (493) de gravidade moderada, $17 \%$ (253) de gravidade menor, 10\% (150) contraindicadas e $5 \%$ (79) de gravidade não especificada. No estudo de Marquito et al. ${ }^{26}$ sobre IM em pacientes renais, a porcentagem de IM de gravidade maior $(16,8 \%)$ foi menos significativa, e a de gravidade moderada $(76,9 \%)$ foi maior do que nos encontrados neste estudo.

Conforme se observa na Tabela 2, as dez IM mais frequentes encontradas neste estudo foram: midazolan $\mathrm{x}$ fentanil com 18,53\%; midazolan x ranitidina 13,05\%; midazolan x omeprazol 11,65\%; ranitidina x fentanila 9,67\%; diazepam x omeprazol 8,15\%; codeína x diazepam 7,45\%; ciprofloxacino e prometazina 6,99\%; metoclopramida x prometazina $6,99 \%$; tramadol $\mathrm{x}$ prometazina 5,94\%; furosemida $\mathrm{x}$ hidralazina $5,47 \%$.

Entre as potenciais IM detectadas, as mais prevalentes, de acordo com a classificação ATC, foram as do grupo $\mathrm{N}$ - medicamentos que agem no sistema nervoso central (SNC) -, destacando-se o benzodiazepínico midazolan e o analgésico opioide fentanil, presentes em 159 prescriçôes. A utilização desses dois medicamentos é bem conhecida na prática clínica, e associada a efeito sinérgico de sedação: terapia empregada na UTI para proporcionar conforto respiratório, alivio da dor e diminuição da ansiedade dos pacientes em ventilação mecânica. Deve-se destacar que essa IM é classificada com gravidade maior por apresentar evento adverso de sedação profunda, devido à hipotensão e à hipoventilação; dessa forma, é importante a constante monitorização do paciente, por exemplo ajustando a dose caso seja necessário ${ }^{13}$. Segundo Devlin et al. ${ }^{27}$, essa pode ser considerada uma IM farmacocinética - já que o fentanil é inibidor do citocromo P4503A4, pelo qual o midazolam é metabolizado - e também farmacodinâmica, pelo efeito sinérgico de sedação. No estudo de Lima et al. ${ }^{22}$, a IM entre midazolam e fentanil foi a mais frequente, representando $14,5 \%$ das IM, assim como no estudo de Reis $^{28}(18,7 \%)$ de um hospital em Belo Horizonte.

A IM entre o midazolam e o antiulceroso ranitidina (grupo A de medicamentos que agem no sistema digestivo) estava presente em 112 prescriçóes. A ranitidina age inibindo o citocromo que metaboliza o midazolam e aumenta sua biodisponibilidade, proporcionando aumento da sedaçãa ${ }^{13}$.

A IM entre o midazolam e o inibidor da bomba de sódio e potássio omeprazol (grupo A de medicamentos que agem no sistema digestivo) estava presente em 100 prescriçóes. O omeprazol é um inibidor do citocromo P4503A4, responsável pela metabolização do midazolam. Dessa forma, com metabolismo reduzido, o benzodiazepínico aumenta seu poder de sedaçáo, tendo como reaçóes adversas letargia e dificuldades na fala. É importante monitorar e ajustar a dose desses medicamentos quando usados concomitantemente ${ }^{13}$.

A IM da ranitidina e do fentanil estava presente em 83 prescrições. A associação do fentanil, um substrato do CYP3A4, ao inibidor do CYP3A4, deve ser feita com bastante cautela, pois pode aumentar os efeitos secundários relacionados ao fentanil, inclusive resultando em depressão respiratória fatal. É necessário monitorar os pacientes que tenham necessidade dessa IM, bem como reduzir a dose caso seja necessário ${ }^{13}$.

A IM do benzodiazepínico diazepam (grupo $\mathrm{N}$ dos medicamentos que agem no SNC) com o omeprazol estava presente em 70 prescriçóes, e pode resultar em um prolongamento do tempo de sedação, visto que o omeprazol é um inibidor do citocromo responsável pela metabolizaçáo do diazepam. É importante monitorar os sinais de sedação excessiva, como letargia, tonturas, ataxia, diminuição da cognição e do desempenho motor. Caso necessário, deve-se realizar a troca do diazepam por um benzodiazepínico que seja eliminado por glucuronidação (lorazepam, oxazepam e temazepam) ${ }^{13}$.

A IM do diazepam e do analgésico opioide codeína (grupo $\mathrm{N}$ dos medicamentos que agem no SNC) foi encontrada em 64 prescrições, e seu resultado é a ocorrência de uma depressão respiratória fatal. Faz-se necessário reduzir a dose de um ou de ambos quando há essa combinação ${ }^{13}$. O emprego de analgésicos na terapia intensiva é 
Tabela 2: Descrição das principais interações medicamentosas nas prescrições dos pacientes internados com suspeita de reações adversas no HGPV de setembro de 2013 a abril de 2014, Jequié (BA).

\begin{tabular}{|c|c|c|c|c|}
\hline Interaçáo medicamentosa & Gestáo clínica & Gravidade & Mecanismo & Fr\% \\
\hline $\begin{array}{l}\text { Midazolan } \mathrm{x} \\
\text { fentanil }\end{array}$ & $\begin{array}{c}\text { Monitorar e ajustar dose se } \\
\text { necessário. }\end{array}$ & Maior & Depressão aditiva do SNC. & 18,53 \\
\hline Midazolan x omeprazol & $\begin{array}{l}\text { Monitorar e/ou substituir } \\
\text { por benzodiazepínico elimi- } \\
\text { nado por glucuronidação. }\end{array}$ & Moderada & $\begin{array}{l}\text { Metabolismo lento do } \\
\text { benzodiazepínico. }\end{array}$ & 11,65 \\
\hline Midazolan x ranitidina & $\begin{array}{l}\text { Monitorar sinais de } \\
\text { intoxicação. }\end{array}$ & Moderada & Diminuição da acidez gástrica. & 13,03 \\
\hline $\begin{array}{l}\text { Ranitidina } \mathrm{x} \\
\text { fentanil }\end{array}$ & $\begin{array}{l}\text { Monitorar observando } \\
\text { depressão respiratória e } \\
\text { sedação excessiva. }\end{array}$ & Maior & $\begin{array}{l}\text { Inibição do metabolismo do fentanil } \\
\text { mediada pelo citocromo P450. }\end{array}$ & 9,67 \\
\hline Diazepam x omeprazol & $\begin{array}{l}\text { Monitorar efeitos excessivos } \\
\text { dos benzodiazepínicos. }\end{array}$ & Menor & Inibição dos metabólitos do diazepam. & 8,15 \\
\hline $\begin{array}{l}\text { Codeína } \mathrm{x} \\
\text { diazepam }\end{array}$ & $\begin{array}{c}\text { Monitorar a depressão } \\
\text { respiratória. }\end{array}$ & Maior & Depressão do SNC. & 7,45 \\
\hline $\begin{array}{l}\text { Ciprofloxacino } \mathrm{x} \\
\text { prometazina }\end{array}$ & $\begin{array}{l}\text { Acompanhar o ECG para } \\
\text { prolongamento de QT. }\end{array}$ & Maior & Efeitos aditivos sobre intervalo de QT. & 6,99 \\
\hline $\begin{array}{l}\text { Metoclopramida } \mathrm{x} \\
\text { prometazina }\end{array}$ & $\begin{array}{l}\text { Monitorar sinais e sintomas } \\
\text { de reaçóes extrapiramidais. }\end{array}$ & Contraindicado & Reação aditiva extrapiramidal. & 6,99 \\
\hline Tramadol x prometazina & $\begin{array}{l}\text { Evitar combinação especial- } \\
\text { mente para paciente com } \\
\text { predisposição a convulsôes. }\end{array}$ & Maior & Desconhecido. & 5,94 \\
\hline Furosemida x hidralazina & $\begin{array}{l}\text { Monitorar eletrólitos } \\
\text { séricos e depuraçáo da } \\
\text { creatinina, se necessário, e } \\
\text { realizar ajuste de dose. }\end{array}$ & Menor & $\begin{array}{l}\text { Aumento da depuração renal da } \\
\text { furosemida. }\end{array}$ & 5,45 \\
\hline
\end{tabular}

Fonte: Pesquisa própria e Micromedex ${ }^{\oplus}$. Fr: frequência

comum, e necessário para conceder um maior conforto ao paciente internado ${ }^{29}$.

A IM do antibiótico ciprofloxacino (grupo J dos antiinfecciosos gerais de uso sistêmico) com o anti-histamínico prometazina (grupo D dos medicamentos dermatológicos) estava presente em 60 prescrições, e pode resultar em aumento do potencial de cardiotoxicidade (prolongamento do intervalo QT). Essa associação deve ser feita com precaução, tendo em vista que idosos são mais sensíveis a esse prolongamento. Se a terapia for necessária, deve-se acompanhar o ECG, obervando o prolongamento do intervalo $\mathrm{QT}^{13}$.

A IM da prometazina com o bloqueador dopaminérgico metoclopramida (grupo A de medicamentos que agem no sistema digestivo) foi encontrada em 60 prescriçóes, e pode resultar em efeitos aditivos de reaçôes extrapiramidais. Se essa terapia for necessária, deve-se monitorar os pacientes quanto ao aparecimento de movimentos involuntários dos membros e reaçóes distônicas, e descontinuar o uso da metoclopramida. A medida adotada para reverter esse quadro é uma injeção de $50 \mathrm{mg}$ de difenidramina ou 1 a $2 \mathrm{mg}$ de benzotropina por via intramuscular ${ }^{13}$.
A IM de prometazina com o analgésico opioide tramadol (grupo $\mathrm{N}$ de medicamentos que agem no SNC) foi observada em 51 prescriçóes e pode aumentar o risco de ocorrência de convulsóes. Deve-se evitar essa associação, principalmente em pacientes que tenham predisposiçáo a convulsóes ${ }^{13}$.

A IM do diurético furosemida (grupo $\mathrm{C}$ de medicamentos que agem no sistema cardiovascular) com o vasodilatador hidralazina (grupo $\mathrm{C}$ de medicamentos que agem no sistema cardiovascular) foi observada em 47 prescriçóes, e pode resultar no aumento da depuração renal da furosemida, porém o mecanismo ainda é desconhecido. Deve-se monitorar os eletrólitos séricos e a depuração da creatinina, realizando o ajuste de dose caso seja necessário ${ }^{13}$.

Nem todas as potenciais IM documentadas na literatura têm seu aparecimento durante a prática clínica; além disso, identificar um evento adverso decorrente de uma IM não é tarefa fácil. Para entender o significado clínico de uma IM é necessária análise minuciosa do paciente, pois, além das patologias associadas, fatores relacionados a medicamentos, idade e sexo atuam como interferentes ${ }^{8}$. Das IM analisadas, 53\% puderam ser observadas clinicamente, das quais $11,08 \%$ 
causaram RAM, conforme mostrado na Figura 1. Reis et al. $^{28}$, em seu estudo sobre fatores associados a IM e aos eventos adversos na UTI, observaram que $34,1 \%$ dos pacientes tiveram RAMs, sendo que $15,5 \%$ delas estavam relacionadas a IM.

Das IM observadas clinicamente, apenas a depressão do SNC não foi considerada uma reação adversa, pois é utilizada com finalidade terapêutica no tratamento intensivo ${ }^{27}$.

Figura 1: Análise das interações medicamentosas dos pacientes com suspeita de reação adversa - 2013-2014, Jequié (BA).

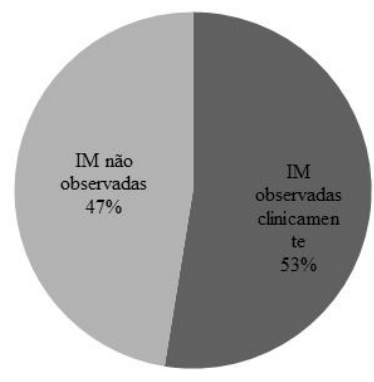

Figura 1a: Frequência relatiobservadas clinicamente.

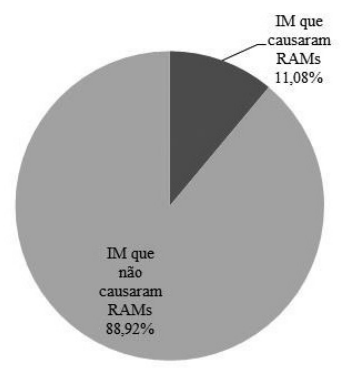

Figura 1c: Distribuição das IM que geraram RAMs. va das IM observadas e não

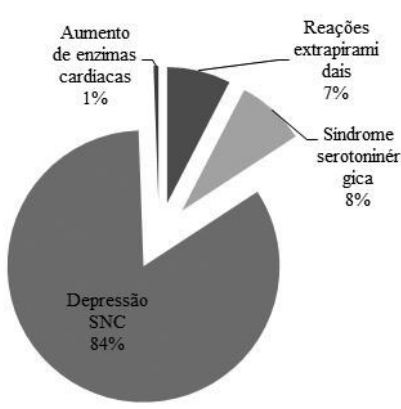

Figura 1b: Frequência relativa das IM observadas clinicamente.

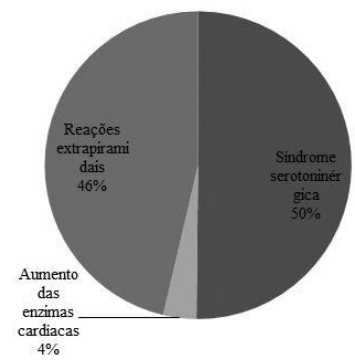

Figura 1d: Distribuição das RAMs geradas por IM.

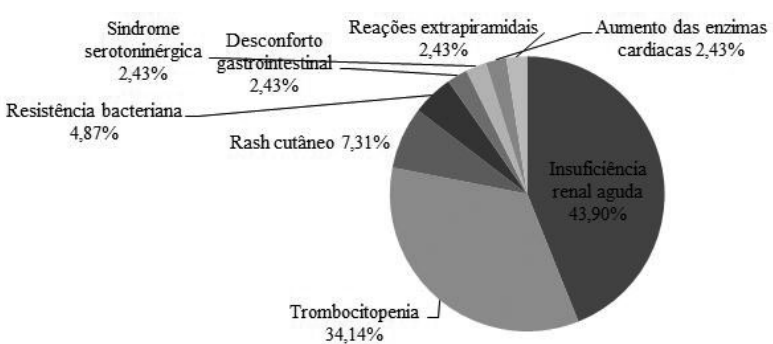

Figura 1e: Frequência das RAMs observadas nos pacientes do estudo.

A potencial IM que pôde ser observada foi entre o antibiótico ciprofloxacino e a dobutamina, uma catecolamina sintética do grupo C (medicamentos que agem no sistema cardiovascular) que foi encontrada em quatro prescriçóes, representando $0,2 \%$ das IM. O uso concomitante desses dois medicamentos pode resultar em aumento do risco de miopatia; essa IM é considerada de gravidade maior. Se essa associaçáo for necessária, deve-se monitorar os níveis das enzimas cardíacas CK (creatina quinase), e interromper o uso se houver aumento acentuado $^{13}$. O paciente em questáo apresentou em seus exames laboratoriais o valor de CK total 657U/L, o que é superior ao valor de referência de 24 a 195 U/L. Outra potencial IM observada foi entre o psicoléptico haloperidol - do grupo $\mathrm{N}$ de medicamentos que agem no SNC - e o bloqueador dopaminérgico metoclopramida, encontrada em 50 prescriçóes, representando 3,31\% das IM. Esse tipo de IM é contraindicado, pois pode resultar em aumento do risco de ocorrência de reaçóes extrapiramidais ou em síndrome neuroléptica maligna; mas, se for necessária, deve-se monitorar o paciente quanto ao aparecimento de sinais e sintomas como febre, acatisia, confusão mental e rigidez muscular. $\mathrm{O}$ paciente em questão apresentou confusão mental e agitação, o que resultou na queda do leito. Lopez et al. ${ }^{29}$, em seu estudo sobre síndrome extrapiramidal induzida por droga, verificou que $40,9 \%$ apresentaram agitação depois do uso de haloperidol e metoclopramida. Outras IM foram observadas no prontuário desse paciente, tais como entre fentanila e antibacteriano linezolida (grupo $\mathrm{J}$ dos anti-infecciosos gerais para uso sistêmico), entre metoclopramida e linezolida, e entre fentanila e amitriptilina, o que representa $7,57 \%$ das IM: todas elas podem resultar no aumento do risco na síndrome serotoninérgica, produção exacerbada de serotonina que é liberada ou permanece no cérebro, causando agitação e alteração da consciência.

Depois de constatada a presença de uma IM, é importante avaliar as condições clínicas e as variáveis que possam determinar sua ocorrência. Neste estudo, observou-se que a probabilidade de ocorrência de IM aumenta em 2,6 vezes para os pacientes que utilizam acima de cinco fármacos, quando comparados àqueles que utilizam até quatro ( $\mathrm{IC}=1,7-3,9)$, conforme tabela 4. Segundo Goldberg et al. ${ }^{30}$, o risco de IM aumenta conforme o número de medicamentos usados, podendo ocorrer em $13 \%$ dos pacientes que utilizam dois medicamentos e em $85 \%$ dos que utilizam mais de seis - ou seja, a polifarmácia é o principal fator de risco para a ocorrência de IM e de eventos adversos a medicamentos ${ }^{31}$. Como se pode observar na tabela 2, do total das RAMs observadas nos pacientes do estudo as mais frequentes foram insuficiência renal $(43,9 \%)$ e trombocitopenia $(34,14 \%)$, com valores acima dos encontrados por Reis ${ }^{28}$, em cujo estudo a nefrotoxicidade representa $16,3 \%$ e a trombocitopenia $2,2 \%$ das RAMs observadas. 
Por meio da análise de variáveis, observou-se que as mais associadas à presença de IM foram idade e sexo: em pacientes acima de 60 anos, a probabilidade de ocorrência de IM é 1,4 vez maior do que em pacientes abaixo disso ( $\mathrm{IC}=1,2-2,4)$; quanto ao sexo, a probabilidade de ocorrência de IM em mulheres é 1,2 vez maior do que em homens ( $\mathrm{IC}=0,8-1,6)$.

Os resultados encontrados neste estudo foram importantes, apresentando um elevado potencial de IM clinicamente relevantes. Diante dos riscos de ocorrência de IM, é pertinente analisar as prescriçôes para ajuste da posologia quando há necessidade de politerapia.

Tabela 3: Odds ratio para ocorrência de interação medicamentosa com sexo, faixa etária e número de medicamentos dos pacientes internados com suspeita de reações adversas no HGPV de setembro de 2013 a abril de 2014, Jequié (BA).

\begin{tabular}{cccccc}
\multicolumn{7}{c}{ Ocorrência de interaçáo medicamentosa } \\
\multicolumn{7}{c}{ Sim } & Não & OR & IC $95 \%$ & $\boldsymbol{P}$-valor \\
No med. \\
Prescrito
\end{tabular}

Fonte: Pesquisa direta.

\section{Conclusão}

Pelos resultados deste estudo, pode-se concluir que o número de medicamentos, a idade e o sexo estão diretamente associados à ocorrência de IM. É importante conhecer as variáveis que estão associadas à ocorrência de IM, seu risco, gravidade e manejo, para minimizar os danos que trazem risco à saúde para proporcionar eficácia, segurança e qualidade na terapêutica.

\section{Referências}

1. Rosholm JU, Bjerrum L, Hallas J, Worm J, Gram LF. Polypharmacy and the risk of drug-drug interactions among Danish elderly: a prescription database study. Dan Med Bull. 1998;45(2):210-3.
2. Brasil. Ministério da Saúde. Secretaria de Tecnologia e Insumos Estratégicos. Departamento de Assistência Farmacêutica e Insumos Estratégicos. Formulário terapêutico nacional. Brasília: Ministério da Saúde, 2008.

3. Jankel CA, Speedie SM. Detecting drug interactions: a review of the literature. DICP. 1990;24(10):982-9.

4. Moura C, Acurcio F, Belo N. Drug-drug interactions associated with length of stay and cost of hospitalization. J Pharm Pharm Sci. 2009;31(5):309-16.

5. Raschetti R, Morgutti M, Menniti-Ippolito F, Belisari A, Rossignoli A, Longhini P, La Guidara C. Suspected adverse drug events requiring emergency department visits or hospital admissions. Eur J Clin Pharmacol. 1999;54(12):959-63.

6. Köhler GI, Bode-Böger SM, Busse R, Hoopmann M, Welte T, Böger RH. Drug-drug interactions in medical patients: effects of in-hospital treatment and relation to multiple drug use. Int J Clin Pharmacol Ther. 2000;38(11):504-13.

7. Gholami K, Shalviri G. Factors associated with preventability, predictability and severity of adverse drug reactions. Ann Pharmacother. 1999;33(2):236-40.

8. Secoli SR. Interaçóes medicamentosas: fundamentos para a prática clínica da enfermagem. Rev Esc Enferm USP. 2001;35(1):28-34.

9. Nies AS. Princípios da terapêutica. In: Hardman JG, Limbird LE, editores. Goodman \& Gilman: as bases farmacológicas da terapêutica. 10 a ed. Rio de Janeiro: McGrawHill; 2003. p. 31-44.

10. Domínguez A. Errores en la medicación: función del farmacêutico. Rev Farm Cuba. 2005;39(2):1-10.

11. Lyra Júnior DP, Kheir N, Abriata JP, Rocha CE, Santos $\mathrm{CB}$, Pelá IR. Impact of pharmaceutical care interventions in the identification and resolution of drug-related problems and on quality of life in a group of elderly outpatients in Ribeirão Preto (SP), Brazil. Ther Clin Risk Manag. 2007;3(6):989-98.

12. Néri E. Determinação do perfil dos erros de prescrição de medicamentos em um hospital universitário [dissertação de mestrado]. Fortaleza: Universidade Federal do Ceará; 2004.

13. Micromedex Healthcare Series 2.0 [base de dados na internet]. Greenwood Village: Thomson Healthcare. Atualizado periodicamente.

14. Organização Mundial da Saúde. CID-10: classificação estatística internacional de doenças e problemas relacionados à saúde. 9a ed. rev. São Paulo: Edusp; 2003.

15. World Health Organization. Collaborating Centre for Drug Statistics Methodology, Guidelines for ATC classification and DDD assignment 2013. Oslo: WHO; 2012.

16. Malone DC, Abarca J, Hansten PD, Grizze AJ, Armstrong EP, Van Bergen RC et al. Identifications of serious drugdrug interactions: results of the partnership to prevent drugdrug interactions. J Am Pharm Assoc. 2004;44(2):142-51.

17. Araujo TG, Rieder MM, Kutchak FM, Franco Filho JW. Readmissóes e óbitos após a alta da UTI: um desafio da terapia intensiva. Rev Bras Ter Intensiva. 2013;25(1):32-8.

18. Melo DO. Avaliação das interaçóes potenciais para pacientes internados na clínica médica do hospital universitário da 
USP visando à elaboração de instrumento para identificação de eventos adversos a medicamentos evitáveis [tese de doutorado]. Sáo Paulo: Faculdade de Ciências Farmacêuticas, Universidade de São Paulo; 2010.

19. Silva MCM, Sousa RMC, Padilha KG. Fatores associados ao óbito e à readmissão em unidade de terapia intensiva. Rev Lat Am Enfermagem. jul-ago 2011;19(4):[9telas].

20. Rossignoli OS, Guarido CF, Cestari IM. Ocorrência de interaçôes medicamentosas em unidade de terapia intensiva: avaliação de prescriçôes médicas. Rev Bras Farm. 2006;87(4): 104-7.

21. Hammes JA, Pfuetzenreiter F, Silveira F, Koenig A, Westpha GA. Prevalência de potenciais interaçóes medicamentosas droga-droga em unidades de terapia intensiva. Rev Bras Ter Intensiva. 2008;20(4):349-54.

22. Lima REF. Interaçóes medicamentosas potenciais em pacientes de unidade de terapia intensiva de um hospital universitário do Ceará [dissertação de mestrado]. Ribeirão Preto: Escola de Enfermagem de Ribeirão Preto, Universidade de São Paulo; 2007.

23. Pincelli MP, Grumann ACB, Fernandes C, Cavalheiro AGC, Haussen DAP, Maia IS. Características de pacientes com DPOC internados em UTI de um hospital de referência para doenças respiratórias no Brasil. J Bras Pneumol. 2011;37(2):217-22.

24. Rama M, Viswanathan G, Acharya LD, Attur RP, Reddy PN, Raghavan SV. Assessment of drug-drug interactions among renal failure patients of nephrology ward in a south indian tertiary care hospital. Indian J Pharm Sci. 2012;74(1):63-8.

25. Reis AM, Cassiani SH. Adverse drug events in an intensive care unit of a university hospital. Eur J Clin Pharmacol. 2011;67(6):625-32.

26. Marquito AB, Fernandes NMS, Colugnatiz FAB, Paula RB. Interaçóes medicamentosas potenciais em pacientes com doença renal crônica. J Bras Nefrol. 2014;36(1):26-34.

27. Devlin JW, Roberts RJ. Pharmacology of commonly used analgesics and sedatives in the ICU: benzodiazepines, propofol, and opioids. Crit Care Clin. 2009;25(3):431-49.

28. Reis AMM. Fatores associados às interaçôes medicamentosas potenciais e aos eventos adversos a medicamentos em uma unidade de terapia intensiva [tese]. Escola de Enfermagem de Ribeirão Preto, Universidade de São Paulo; 2009.

29. Lopez RF, Couce PM, Calvo FJ, Castro-Gaco M. Drug-induced extra-pyramidal syndrome. QJ Med. 1986;59(230):549-56.

30. Goldberg RM, Mabee J, Chan L, Wong S. Drugdrug and drug-disease interactions in the ED: analysis of a high-risk population. Am J Emerg Med. 1996; 14(5):447-50.

31. Locatelli J. Interaçôes medicamentosas em idosos hospitalizados. Einstein. 2007;5(4):343-6.

\section{Como citar este artigo:}

Gonçalves SS, Rodrigues HMS, Jesus IS, Carneiro JAO, Lemos GS. Ocorrência clínica de interaçóes medicamentosas em prescriçóes de pacientes com suspeita de reação adversa internados em um hospital no interior da Bahia. Rev. Aten. Saúde. 2016;14(48):32-39. 